\title{
Application of BOLD-MRI in the classification of renal function in chronic kidney disease
}

\author{
Chenxia Li, ${ }^{1,2}$ Haitian Liu, ${ }^{1}$ Xiang Li, ${ }^{1}$ Li Zhou, ${ }^{1}$ Rong Wang, ${ }^{1}$ and Yuelang Zhang ${ }^{1}$ \\ ${ }^{1}$ Department of Radiology, The First Affiliated Hospital of Xi'an Jiaotong University, 277 West Yanta Road, Xi'an, Shaanxi \\ Province, China \\ ${ }^{2}$ Department of Biomedical Engineering, The Key Laboratory of Biomedical Information Engineering of the Ministry of Education, \\ School of Life Science and Technology, Xi'an Jiaotong University, Xi'an, China
}

\section{Abstract}

Purpose: The purpose of the study was to explore the application of blood oxygenation level-dependent functional magnetic resonance imaging (BOLD-MRI) in classification of chronic kidney disease (CKD).

Methods: Twenty-nine cases with CKD and 27 healthy volunteers underwent renal BOLD-MRI. Cases of CKD were divided into two groups according to the estimated glomerular filtration rate (eGFR). The R2* values were measured in renal cortex and medulla, respectively. The difference of R2* between renal cortex and medulla was compared, and the correlations of $\mathrm{R} 2 *$ value in renal cortex and medulla with eGFR were analyzed.

Results: Twenty-nine cases of CKD were divided into two groups, with 13 cases of mild renal impairment and 16 cases of moderate to severe renal impairment. In the control and mild renal impairment group, the R2* of renal cortex was significantly lower than that of medulla $(P<0.001)$. In the control group, mild renal impairment and moderate to severe renal impairment group, the R2* value of cortex increased, while the R2* value of medulla gradually decreased. The eGFR of patients was positively correlated with R2* of medulla $(r=0.81$, $P<0.001)$, while displayed no correlation with R2* of cortex $(r=-0.32, P>0.05)$. When the threshold of R2* of medulla was set at $28.4 \mathrm{~Hz}$, the sensitivity and specificity to distinguish normal and mild renal impairment group were $92.31 \%$ and $85.19 \%$, respectively.

Conclusion: The change of blood oxygen in renal cortex and medulla could be detected with BOLD-MRI, so as to evaluate the renal function and anoxic injury of CKD.

Chenxia Li and Haitian Liu have contributed equally to this work and should be considered co-first authors.

Correspondence to: Yuelang Zhang; email: 18991232590@163.com
Key words: Blood oxygenation leveldependent-Magnetic resonance imaging - Chronic kidney disease-Renal function- $\mathrm{R} 2 *$

Chronic kidney disease (CKD) shows gradually increased incidence and prevalence recently, thus becoming a public health problem [1]. Glomerular injury is a main pathological mechanism of CKD and could change local hemodynamics, damage capillary endothelial and microvasculature, thereby leading to anoxic tissue environment and kidney damage [2]. Hypoxia in kidney tissue will accelerate the progress of CKD and play a key role in its occurrence and development. Therefore, early monitoring of renal oxygenation and reasonable treatment could help delay the progression of CKD [3, 4]. The current common method to determine renal hypoxia is to measure the partial pressure of oxygen by inserting microelectrode into the kidneys directly. However, this technology is not suitable for in vivo research and followup due to its invasiveness and insufficient sampling [5].

Blood oxygenation level-dependent functional magnetic resonance imaging (BOLD-MRI) is the only noninvasive technology to perform in vivo imaging on renal oxygen content. The technique uses endogenous deoxyhemoglobin as contrast agent to evaluate the biological oxygen utilization [6]. Pedersen et al. performed animal experiments and have confirmed that BOLD could detect renal oxygenation levels sensitively, which was highly consistent with the data measured by invasive probes [713]. The content of oxygenated hemoglobin is inversely proportional to the apparent relaxation rate $(\mathrm{R} 2 *)$ obtained by BOLD. The higher the tissue oxygen content, the lower the R2* value is. Therefore, R2* can reflect the partial pressure of oxygen in local tissue indirectly [8]. This study aimed to analyze the correlation between R2* 
values and estimated glomerular filtration rate (eGFR), and to investigate the application value of BOLD-MRI in assessment renal oxygen level of patients with CKD.

\section{Materials and methods}

\section{Subjects}

The protocol of this study was discussed, approved and recorded by the ethics committee of our institution. A total of 29 patients with CKD were prospectively collected from December 2015 to June 2016 in Department of nephrology of our hospital, with the average age of 42 years old (23-63). Twenty-seven healthy volunteers were selected as the control group, including 16 males and 11 females, with the average age of 39 years old (18-59). Informed consent was obtained from all participants included in this study.

\section{The inclusion and exclusion criteria of CKD}

The inclusion criteria were as follows: (1) more than 18 years old; (2) in accordance with the CKD definition from the kidney disease outcomes quality initiative $(\mathrm{K} /$ DOQI) proposed by National Kidney Foundation [14]; (3) without contraindications of MRI examination. Exclusion criteria were as follows: (1) complicated with other kidney diseases such as kidney stones, hydronephrosis, renal mass, polycystic kidney disease, and other systemic diseases; (2) taking the drugs that affect the measurement value of creatinine (such as cimetidine, trimethoprim, cefotaxime) or undergoing renal replacement therapy; (3) difficult to be analyzed because of poor image quality.

\section{The inclusion and exclusion criteria of healthy controls}

The inclusion criteria were as follows: (1) more than 18 years old; (2) without urinary system disease, systemic metabolic or endocrine diseases, diabetes mellitus or hypertension; (3) laboratory tests related to renal function were normal; (4) no contraindications to MRI. Exclusion criteria were as follows: (1) renal space-occupying lesions confirmed by MRI, hydronephrosis and infectious lesions; (2) vascular and nephrotoxic drugs were used within 3 months before the examination; (3) difficult to be analyzed because of poor image quality.

\section{MRI protocol}

All the scan were performed on a 3.0T magnetic resonance scanner (Signa HDxt, General Electric Medical System, Milwaukee, WI, USA) with 8-channel phased array coils for the body. All the participants were performed BOLD-MRI examination with routine T1WI and T2WI scans. Sequence of BOLD was two-dimen- sional (2D) multiple echoes gradient echo (mGRE), including a total of 7 echoes. The coronal scan was performed and the scan parameters were as follows: TR: $100 \mathrm{~ms}$, TE:1.76, 4.18, 6.6, 9.01, 11.43, 13.84, 16.26, $18.68 \mathrm{~ms}$, thickness: $5 \mathrm{~mm}$, spacing: $0 \mathrm{~mm}$, flip angle: 25 degrees, bandwidth: $125 \mathrm{kHz}$, excitation frequency: 1, FOV: $36-42 \mathrm{~cm}$, matrix: $256 \times 256$, the coverage of scanning included left and right kidneys and adrenal gland. All the subjects were fasting and avoided medicine 6 hours before MRI scan.

\section{Image analysis and data measurement}

After these sequences were performed, the images were transmitted to the AW4.4 workstation, and the data were analyzed by Functool post-processing software. Based on the single exponential model, R2* value was obtained by fitting calculation of echo signal and TE time. At the coronal central level of renal hilum, three locations were selected from the upper pole, the renal portal and the inferior pole. The region of interest (ROI) was placed in cortex and medulla, respectively, with the area about $10-20 \mathrm{~mm}^{2}$, avoiding the renal collection system. The R2* values of renal cortex and medulla of each subject were averaged from left and right kidneys, cortex $\mathrm{R} 2^{*}=$ (right renal cortex $\mathrm{R} 2 *+$ left renal cortex $\left.\mathrm{R} 2 *\right)$ / 2 , and medulla $\mathrm{R} 2 *=$ (right renal medulla $\mathrm{R} 2 *+$ left renal medulla $\mathrm{R} 2 *) / 2$. Twice measurements were performed on the kidneys by the same radiologist, and the interval between the two measurements was 30 days.

\section{Assessment of glomerular filtration rate}

For all patients, venous blood were collected 1 week before and after MRI examination, respectively. Serum creatinine (Scr) levels were measured and expressed as $\mathrm{mg} /$ $\mathrm{dL}$ from the conversion formula: $1 \mathrm{mg} / \mathrm{dL}=88.41 \mu \mathrm{mol} /$ L. The eGFR was obtained from the modification of diet in renal disease (MDRD) equation. eGFR $=186 \times \mathrm{Scr}^{-}$ $1.154 \times \mathrm{Age}^{-0.203} \times 0.742$ (if female) $\times 1.233$ (if Chinese)

According to the K/DOQI guideline, CKD clinical stage was divided by the degree of renal impairment based on eGFR. CKD1: eGFR $\geq 90 \mathrm{~mL} / \mathrm{min}$; CKD2: $90 \mathrm{~mL} / \mathrm{min}>\mathrm{eGFR} \geq 60 \mathrm{~mL} / \mathrm{min} ; \quad$ CKD3: $60 \mathrm{~mL} /$ $\min >\mathrm{eGFR} \geq 30 \mathrm{~mL} / \mathrm{min} ; \quad \mathrm{CKD} 4: \quad 30 \mathrm{~mL} / \mathrm{min}>$ eGFR $\geq 15 \mathrm{~mL} / \mathrm{min} ; \quad$ CKD5: $\quad$ eGFR $<15 \mathrm{~mL} / \mathrm{min}$. Cases of CKD 1 and 2 were assigned to mild renal impairment group. Cases of CKD 3, 4, and 5 were assigned to moderate to severe renal impairment group.

\section{Statistical analysis}

Data were analyzed by SPSS 21.0 statistical software (SPSS, Inc, Chicago, IL, USA). First, consistency test was performed by calculating the intraclass correlation coefficient (ICC) of two measurement data. If ICC was 
greater than 0.75 , a high reliability was considered and the first measurement data were used. If normal distribution of the data was confirmed, paired $t$ test was used to compare the difference in renal cortex and medulla R2* between left and right side. Independent samples $t$ test was used to compare the difference of R2* between cortex and medulla in each group. When the data complied with the normal distribution and the homogeneous variance, the single factor analysis of variance (one-way ANOVA) was used to compare the R2* difference of cortex and medulla among the three groups, with mutual comparison of each group by least-significant difference (LSD) method. Bivariate correlation analysis was performed to calculate Pearson correlation coefficient, so as to evaluate the correlation of eGFR with cortex and medulla R2* in patients with CKD. The receiver operating characteristic (ROC) curve was analyzed by MedCalc software (version 13.0) to distinguish normal and mild renal impairment group. The significant level of $\alpha=0.05$ was tested, and $P<0.05$ was considered as statistically significant, and $P<0.001$ was considered as highly statistical significance.

\section{Results}

\section{Clinical and pathological results}

The clinical biochemical data of healthy controls and the patients group are shown in Table 1. According to the renal function evaluated by eGFR, 29 cases of CKD were divided into two groups: 13 cases of mild renal impairment group (CKD1 and CKD2) and 16 cases of moderate to severe renal impairment group (CKD3, CKD4, and CKD5). Twenty-three cases of CKD patients received renal tissue biopsy under ultrasound guidance, with the lower pole of the right kidney as puncture point. Nine cases were diagnosed as membranous nephropathy, nine cases as IgA nephropathy, two cases as focal proliferative sclerosing glomerulonephritis, and three cases as mesangial proliferative glomerulonephritis. The other six cases without renal biopsy were all in the stage of CKD5.

\section{Normal kidney BOLD-MRI image and data consistency analysis}

On the BOLD-MRI image, the normal renal structure was clear, with distinct interface between cortex and medulla. From renal cortex to medulla, the R2* images displayed gradually from blue, green, to yellow (Fig. 1). Data consistency analysis showed that the intraclass correlation coefficient of R2* was $98.5 \%$ $(\mathrm{ICC}>0.75)$ for cortex and $99.8 \%(\mathrm{ICC}>0.75)$ for medulla.

\section{Comparison of $R 2^{*}$ values between renal cortex and medulla}

Paired $t$ test showed that there was no significant difference in $\mathrm{R} 2 *$ value of cortex and medulla between left and right kidney in the 3 groups (Table 2). Independent sample $t$ test showed that the R2* values of medulla were significantly higher than those of cortex in healthy control group and mild renal impairment group $(P<0.001)$. There was no difference in $\mathrm{R} 2 *$ between medulla and cortex in moderate to severe renal impairment group $(P>0.05)$ (Table 3$)$.

\section{Comparison of $R 2^{*}$ values among three groups}

Univariate analysis of variance showed that there were statistically significant differences in R2* values of renal cortex and medulla among control group, mild renal impairment group and moderate to severe renal impairment group $(P<0.001)$ (Table 3$)$. LSD comparison showed that the R2* in cortex gradually increased from control group, mild renal impairment group, to moderate to severe renal impairment group, with statistical difference $(P<0.05)$ (Fig. 2$)$. The R2* of medulla in the control group, mild renal impairment group and moderate to severe renal impairment group gradually decreased, with statistical difference $(P<0.001)$ (Fig. 2).

Table 1. Basic information of healthy volunteers and patients with chronic kidney disease

\begin{tabular}{lccr}
\hline Class & $N$ & Age & eGFR \\
\hline Healthy volunteers & 27 & $39.69 \pm 14.88$ & $110.79 \pm 11.17$ \\
CKD1 & 8 & $42.50 \pm 14.74$ & $118.13 \pm 20.15$ \\
eGFR $\geq 90 \mathrm{~mL} / \mathrm{min} / 1.73 \mathrm{~m}^{2}$ & 5 & $48.20 \pm 14.36$ & $73.74 \pm 7.57$ \\
$\mathrm{CKD} 2$ & 6 & $48.00 \pm 12.46$ & $43.08 \pm 6.99$ \\
$60 \mathrm{~mL} / \mathrm{min} / 1.73 \leq \mathrm{eGFR}<90 \mathrm{~mL} / \mathrm{min} / 1.73 \mathrm{~m}^{2}$ & 3 & $44.66 \pm 6.66$ & $21.33 \pm 6.11$ \\
$\mathrm{CKD} 3$ & $3 \mathrm{~mL} / \mathrm{min} / 1.73 \mathrm{~m}^{2} \leq \mathrm{eGFR}<60 \mathrm{~mL} / \mathrm{min} / 1.73 \mathrm{~m}^{2}$ & $33.57 \pm 11.67$ & $9.73 \pm 4.10$ \\
$\mathrm{CKD} 4$ & 7 & & \\
$15 \mathrm{~mL} / \mathrm{min} / 1.73 \mathrm{~m}^{2} \leq \mathrm{eGFR}<30 \mathrm{~mL} / \mathrm{min} / 1.73 \mathrm{~m}^{2}$ & & & \\
eKD & & & \\
\hline
\end{tabular}



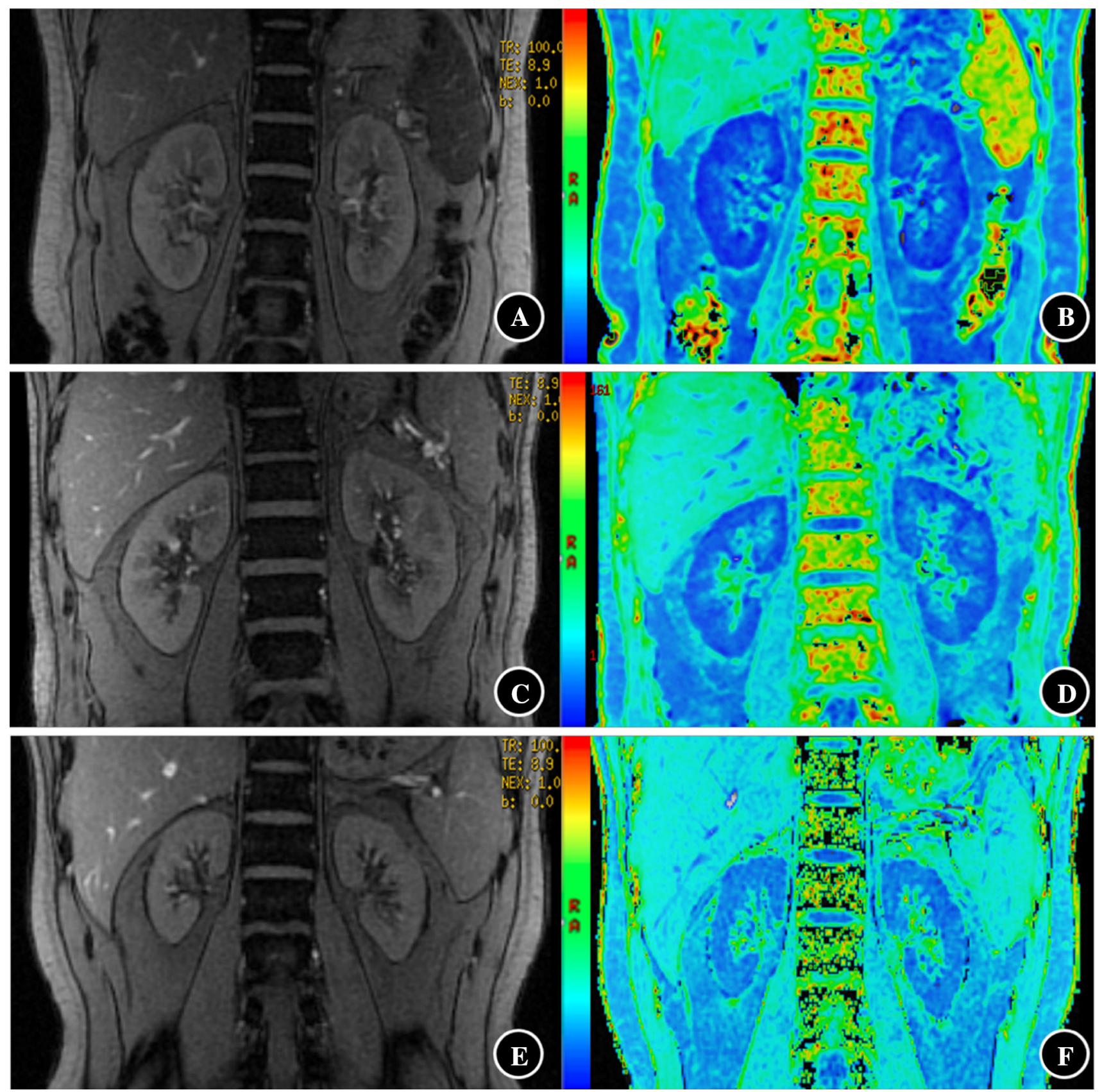

Fig. 1. A, B A 18-year-old male of normal control group, $\mathrm{T} 2^{*} \mathrm{WI}$ and $\mathrm{R} 2^{*}$ map in coronal plane. The demarcation of the renal cortex and medulla is clear. The color of the cortex, medulla, and renal calyx is transformed from blue, green to yellow. The color change from blue to green and yellow represents the gradual increase of R2*.C, D A 38-year-old male with IgA glomerulonephritis of mild renal impairment group, $\mathrm{T}^{*} \mathrm{WI}$ and $\mathrm{R} 2^{*}$ map in coronal plane. The demarcation of the renal cortex and medulla is slightly clear; the cortex is

The correlation of $R 2 *$ value with eGFR and $C K D$ staging

The Pearson correlation analysis was performed to analyze the relationship of $\mathrm{R} 2 *$ value and eGFR. It blue, and the medulla is green. E, F A 29-year-old male with IgA glomerulonephritis of moderate to severe renal impairment group, $\mathrm{T}^{*} \mathrm{Wl}$ and $\mathrm{R}^{*}$ map in coronal plane. There is unclear boundary between the renal cortex and medulla. The cortex is blue to green and the medulla is also blue to green. The color of the cortex changed from blue to green with the aggravation of renal impairment gradually. The color of medulla changed from green-yellow to blue with the severity of renal impairment gradually.

showed that in all CKD patients there was no statistical correlation between cortical R2* value and eGFR $(r=-0.319, P>0.05)$. However, medullary R2* value showed statistical positive correlation with eGFR $(r=0.806, P<0.001)$ (Fig. 3$)$. There was slight posi- 
Table 2. Comparison of cortical and medullary R2* between left and right kidneys

\begin{tabular}{|c|c|c|c|c|c|c|}
\hline \multirow[t]{2}{*}{ Goup } & \multicolumn{2}{|c|}{ Cortical R2* } & \multirow[t]{2}{*}{$P$} & \multicolumn{2}{|c|}{ Medullary R2* } & \multirow[t]{2}{*}{$P$} \\
\hline & Left & Right & & Left & Right & \\
\hline Control & $13.15 \pm 0.74$ & $13.25 \pm 1.00$ & 0.59 & $32.62 \pm 4.26$ & $32.32 \pm 4.59$ & 0.51 \\
\hline Mild & $16.15 \pm 1.10$ & $16.35 \pm 1.02$ & 0.55 & $25.45 \pm 3.63$ & $24.35 \pm 2.83$ & 0.06 \\
\hline Moderate to severe & $16.77 \pm 1.09$ & $17.16 \pm 1.39$ & 0.27 & $18.03 \pm 3.15$ & $18.06 \pm 3.21$ & 0.91 \\
\hline
\end{tabular}

Table 3. Comparison of cortical and medullary R2* values in the three groups

\begin{tabular}{|c|c|c|c|c|c|}
\hline $\mathrm{R} 2 *$ & Control $(n=27)$ & Mild $(n=13)$ & Moderate to severe $(n=16)$ & $F$ & $P$ \\
\hline Cortex & $13.20 \pm 0.75$ & $16.25 \pm 0.89$ & $16.97 \pm 1.05$ & 115.87 & $<0.05$ \\
\hline Medulla & $32.47 \pm 4.28$ & $24.90 \pm 3.11$ & $18.04 \pm 3.142$ & 76.44 & $<0.05$ \\
\hline $\mathrm{t}$ & -23.02 & -9.64 & -1.30 & - & - \\
\hline $\mathrm{P}$ & $<0.05$ & $<0.05$ & 0.21 & - & - \\
\hline
\end{tabular}

A

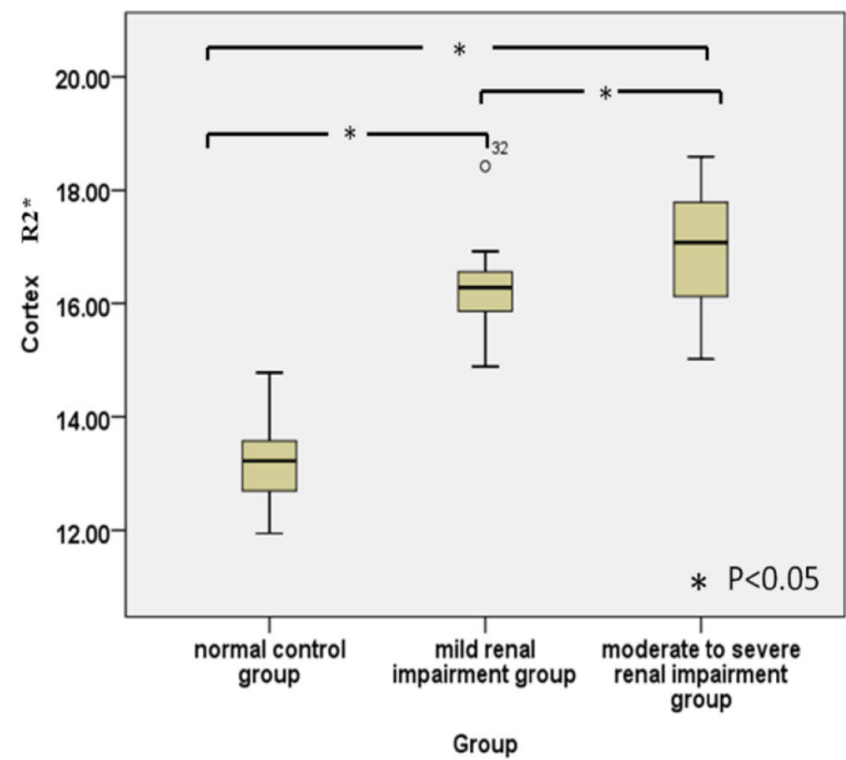

B

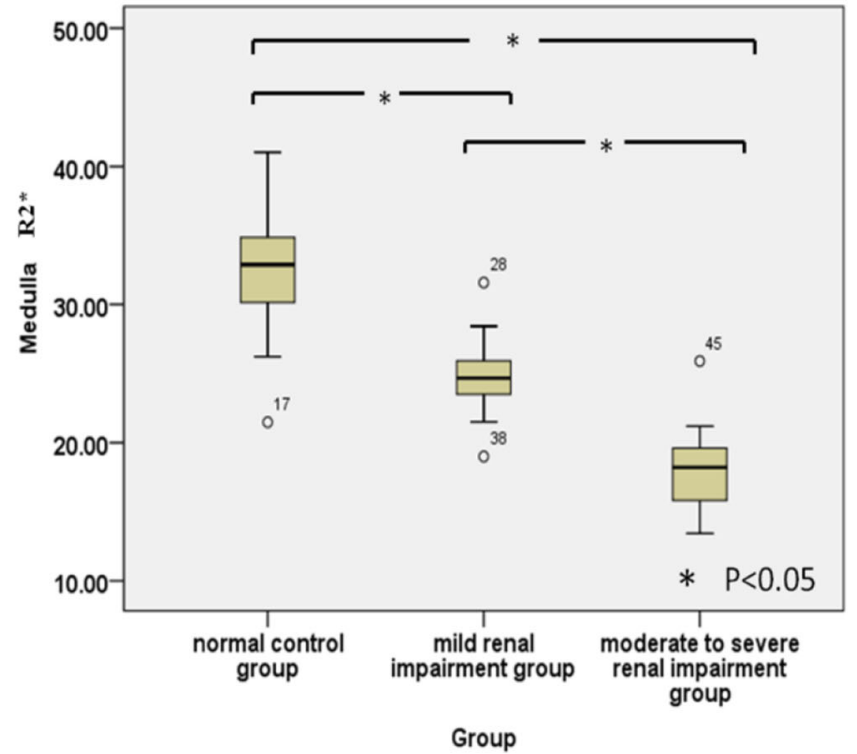

Fig. 2. Comparison of cortical (A) and medullary (B) R2* values among normal control group, mild renal impairment group, and moderate to severe renal impairment group.

tive correlation between cortical R2* value and CKD stage $(r=0.379, P<0.05)$, and significant negative correlation between the medullary R2* and CKD stage $(r=-0.825, P<0.001)$.

\section{ROC analysis of medullary $R 2^{*}$ to distinguish normal from early renal impairment}

In the normal control group, mild renal impairment group and moderate to severe renal impairment group, medullary R2* showed more difference among three groups than cortical R2* did. Therefore, the medullary $\mathrm{R} 2 *$ value was used to evaluate its diagnostic efficiency to distinguish healthy control and mild renal impairment group. The ROC analysis showed that the area under the curve (AUC) was 0.892 (Fig. 4). The optimal diagnostic threshold of medullary R2* was $28.426 \mathrm{~Hz}$ by calculating the Youden index, and the sensitivity and specificity for distinguishing normal from mild renal impairment were $92.31 \%$ and $85.19 \%$, respectively.

\section{Discussion}

The results of this study showed that there was favorable intra-observer consistency of cortical and medullary R2*. It displayed significant difference in renal cortical and medullary R2* values among healthy control, mild renal impairment group and moderate to severe renal impairment group. There was significant positive correlation between eGFR and R2* values of medulla. All 

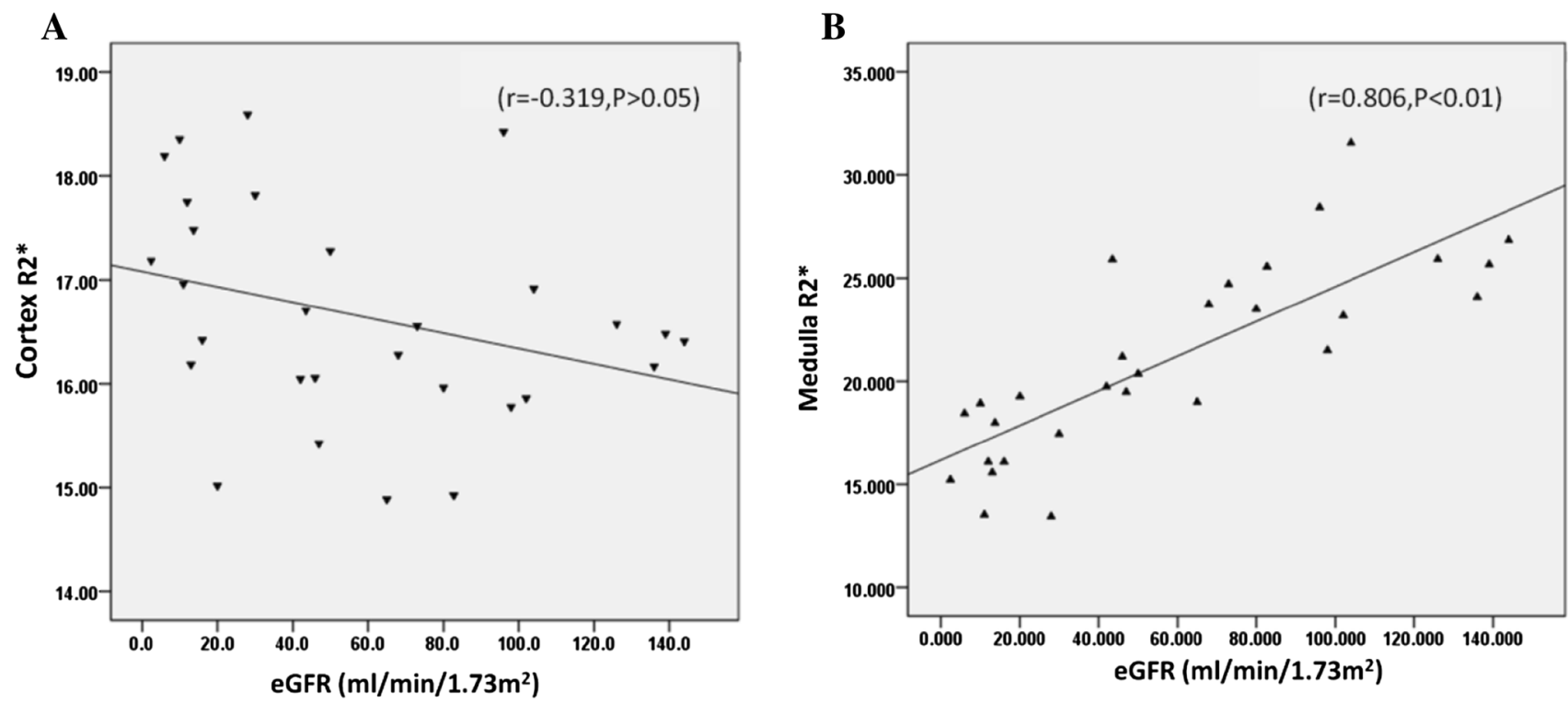

Fig. 3. The correlation between $R 2^{\star}$ value of cortex $(\mathbf{A})$ and medulla $(\mathbf{B})$ with eGFR.

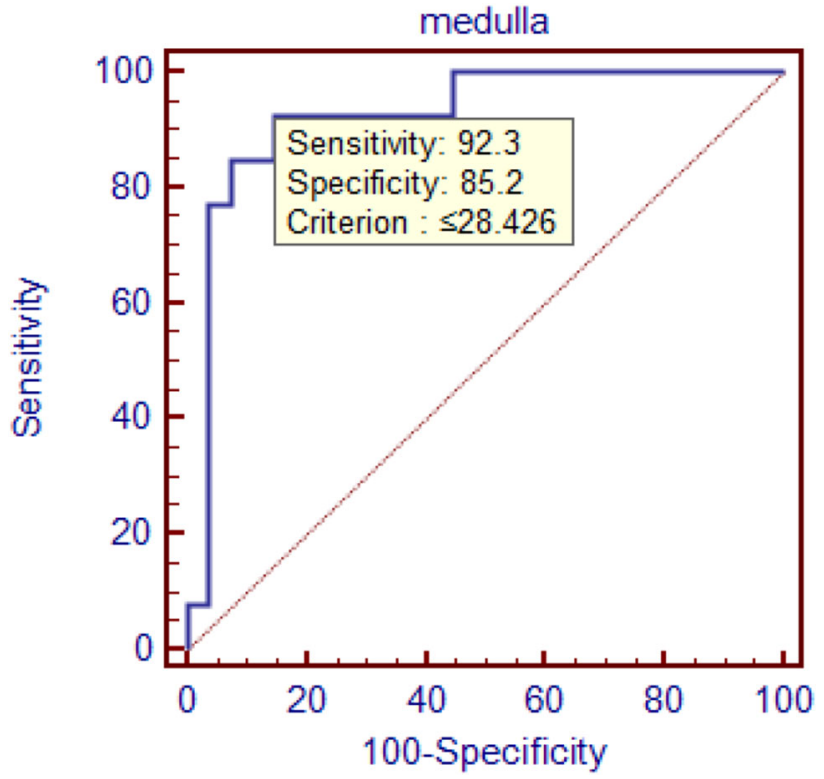

Fig. 4. The ROC curve of medullary $R 2^{*}$ value to differentiate normal control group from the mild renal impairment group.

these suggested that BOLD-MRI technique was sensitive to hypoxia in early renal impairment, and had certain feasibility and data repeatability.

In healthy control group, the R2* value of renal medulla was significantly higher than that of cortex. This indicated that the oxygen content of medulla was significantly lower than that of cortex, which was consistent with renal physiology and the difference of blood distribution between medulla and cortex. It is known that the cortex accounts for $94 \%$ blood flow perfusion of kidney, which is markedly higher than that of medulla.
The R2* of medulla was significantly greater than that of cortex in the mild renal impairment group, while there was no significant difference in R2* between medulla and cortex in the moderate to severe renal impairment group. This suggested that renal oxygenation was not obviously affected in the early stage of renal impairment, which may be adjusted by compensative effect of kidney. However, in moderate to severe renal impairment group, the blood flow and oxygenation status of cortex and medulla were all seriously injured. The content of deoxyhemoglobin in both groups increased significantly, and the difference was attenuated or even disappeared.

The R2* value of cortex in CKD cases was significantly higher than that of the normal control group. This suggested that the renal cortex of the patients group was in a relatively hypoxic state, with positive correlation between degree of hypoxia and renal impairment, which was consistent with the results of other researchers [1517]. The underlying mechanism might be associated with capillary lumen stenosis and occlusion caused by deposition of plasma protein in renal cortex, thickening of the basement membrane, and increased membrane matrix with the progression of CKD. Furthermore, increased extracellular collagen in mesangial and capillary loops could lead to local capillary lumen occlusion, capillary hyalinization and sclerosis. This further accelerated glomerular hardening and reduced blood flow, so the oxygen content of cortex decreased significantly [18].

The R2* value of medulla in the normal control group, mild renal impairment group and moderate to severe renal impairment group gradually decreased, with significant differences between each two groups. This suggested that with the decline of renal function in patients group, the content of deoxygenated hemoglobin in medulla gradually decreased and the level of oxygenation 
gradually increased. This was not entirely consistent with the results of other researchers. Prasad PV found that there was no significant difference in $\mathrm{R} 2 *$ values of medulla between normal control group and CKD cases [17]. Michaely HJ observed 400 cases of various abdominal diseases and found that medullary R2* did not display significant difference among patients with different stages of CKD. This might because there was no unified standard for inclusion of patients and control group among different studies [19]. The results of our study suggested that with the decline of renal function, the oxygen content in the medulla increased gradually. We speculated the possible reason as follows: as the renal function impairment worsens, not only the glomerular filtration rate decreases, but also ultrafiltration is further reduced. That attenuated active absorption of $\mathrm{NaCl}$ in the medulla proximal tubule. At the same time, CKD accompanied by varying degrees of tubular atrophy, the reduction of active transport of small molecules and tubular atrophy together cause $\mathrm{Na}^{+}-\mathrm{K}^{+}$-ATP pump work reduction and oxygen consumption decrease [20]. Khatir D found that in patients with CKD stage 3 and 4, the sodium ion reabsorption rate was only $37 \%$ of that in normal people. Glomerular filtration rate and sodium reabsorption decreased more than twofolds of renal artery blood flow. The decreased filtration fraction reduced $\mathrm{Na}^{+}$Pump work, thus alleviating the hypoxia of the renal medulla [21].

In patients with CKD, there was a significant positive correlation between the R2* value of medulla and eGFR, while no correlation was found between $\mathrm{R} 2$ * value of renal cortex and eGFR. This might be caused by the difference between medulla and cortex in distribution of renal oxygen pressure and their sensitivity to the change of oxygen status. Under normal conditions, the partial pressure of oxygen is about $50 \mathrm{mmHg}$ in cortex, and about $10 \mathrm{mmHg}$ in medulla [22, 23]. The oxygen dissociation curve becomes flat when the partial pressure of oxygen is above $60 \mathrm{mmHg}$, and becomes steeper when the partial pressure of oxygen is below $26.6 \mathrm{mmHg}$. This indicated that even a small change in oxygen partial pressure in medulla will lead to a huge change of deoxyhemoglobin concentration and subsequently R2* value. Therefore, we further selected medullary $\mathrm{R} 2 *$ to detect its ability to distinguish the severity of renal function. The result showed that it displayed satisfactory sensitivity and specificity $92.31 \%$ and $85.19 \%$, respectively. This suggests that R2* obtained by BOLD-MRI technology could reflect the change of renal oxygenation level by measuring the concentration of deoxyhemoglobin, and further evaluate the renal function.

The limitations of this study were as follows: (1) The sample size in this study was not large enough, and most CKD cases were glomerular nephropathy. Though CKD in Chinese patients is mainly caused by glomerular lesion, further clinical research is needed to investigate the difference in R2* value among CKD caused by different etiologies. (2) With the progression of CKD stage, the unclear boundary between medulla and cortex might influence the accuracy of measurement. Therefore, we carried out the intra-observer consistency test, so as to reduce the variance of measurement. (3) Compared with control group, the patients with CKD demonstrate smaller renal volume and lower renal plasma flow, which might affect the results of the experiment. However, it was also reported that when the renal plasma flow was corrected, there was no difference in $\mathrm{R} 2 *$ value between CKD group and normal control group [21].

In conclusion, this study displayed that BOLD-MRI could detect the oxygenation level of renal cortex and medulla at different levels of renal impairment, and also provided information about renal morphology and function. It is expected that BOLD-MRI might provide a sensitive, noninvasive and reproducible method for detection of possible hypoxia in the renal parenchyma and assessment of renal function.

Acknowledgements This study was supported by the Clinical Research Award of the First Affiliated Hospital of Xi'an Jiaotong University, China (No. XJTU1AHCR2014-037).

Compliance with ethical standards

Funding The study was funded by No. XJTU1AHCR2014-037.

Conflict of interest All the authors declare no conflicts of interest.

Ethical approval All procedures performed in this study were in according with the ethical standards of the institutional and/or national research committee and with the 1964 Helsinki declaration and its later amendments or comparable ethical standards.

Informed consent Informed consent was obtained from all individual participants included in the study.

Open Access This article is distributed under the terms of the Creative Commons Attribution 4.0 International License (http://creativecom mons.org/licenses/by/4.0/), which permits unrestricted use, distribution, and reproduction in any medium, provided you give appropriate credit to the original author(s) and the source, provide a link to the Creative Commons license, and indicate if changes were made.

\section{References}

1. Hunsicker LG (2004) The consequences and costs of chronic kidney disease before ESRD. J Am Soc Nephrol 15(5):1363-1364

2. Fine LG, Norman JT (2008) Chronic hypoxia as a mechanism of progression of chronic kidney diseases: from hypothesis to novel therapeutics. Kidney Int 74(7):867-872. https://doi.org/10.1038/ki. 2008.350

3. Nangaku M (2004) Hypoxia and tubulointerstitial injury: a final common pathway to end-stage renal failure. Nephron Exp Nephrol 98(1):e8-12. https://doi.org/10.1159/000079927

4. Palm F, Nordquist L (2011) Renal tubulointerstitial hypoxia: cause and consequence of kidney dysfunction. Clin Exp Pharmacol Physiol 38(7):474-480. https://doi.org/10.1111/j.1440-1681.2011.05 532. $\mathrm{x}$

5. Heyman SN, Khamaisi M, Rosen S, Rosenberger C (2008) Renal parenchymal hypoxia, hypoxia response and the progression of chronic kidney disease. Am J Nephrol 28(6):998-1006. https://doi. org $/ 10.1159 / 000146075$ 
6. Lanzman RS, Notohamiprodjo M, Wittsack HJ (2015) Functional magnetic resonance imaging of the kidneys. Der Radiol 55(12):1077-1087. https://doi.org/10.1007/s00117-015-0044-z

7. Pedersen M, Dissing TH, Morkenborg J, et al. (2005) Validation of quantitative BOLD MRI measurements in kidney: application to unilateral ureteral obstruction. Kidney Int 67(6):2305-2312. http s://doi.org/10.1111/j.1523-1755.2005.00334.x

8. Tumkur SM, Vu AT, Li LP, Pierchala L, Prasad PV (2006) Evaluation of intra-renal oxygenation during water diuresis: a timeresolved study using BOLD MRI. Kidney Int 70(1):139-143. http s://doi.org/10.1038/sj.ki.5000347

9. dos Santos EA, Li LP, Ji L, Prasad PV (2007) Early changes with diabetes in renal medullary hemodynamics as evaluated by fiberoptic probes and BOLD magnetic resonance imaging. Investig Radiol 42(3):157-162. https://doi.org/10.1097/01.rli.0000252492.96 709.36

10. Li LP, Ji L, Santos E, et al. (2009) Effect of nitric oxide synthase inhibition on intrarenal oxygenation as evaluated by blood oxygenation level-dependent magnetic resonance imaging. Investig Radiol 44(2):67-73. https://doi.org/10.1097/RLI.0b013e318190097 5

11. Warner L, Glockner JF, Woollard J, et al. (2011) Determinations of renal cortical and medullary oxygenation using blood oxygen level-dependent magnetic resonance imaging and selective diuretics. Investig Radiol 46(1):41-47. https://doi.org/10.1097/RLI.0b013e31 $81 \mathrm{f0213f}$

12. Pruijm M, Hofmann L, Piskunowicz M, et al. (2014) Determinants of renal tissue oxygenation as measured with BOLD-MRI in chronic kidney disease and hypertension in humans. PloS ONE 9(4):e95895. https://doi.org/10.1371/journal.pone.0095895

13. Zhang JL, Morrell G, Rusinek H, et al. (2014) Measurement of renal tissue oxygenation with blood oxygen level-dependent MRI and oxygen transit modeling. Am J Physiol Renal Physiol 306(6):F579-587. https://doi.org/10.1152/ajprenal.00575.2013

14. National Kidney F (2002) K/DOQI clinical practice guidelines for chronic kidney disease: evaluation, classification, and stratification. Am J Kidney Dis 39(2 Suppl 1):S1-266
15. Inoue T, Kozawa E, Okada H, et al. (2011) Noninvasive evaluation of kidney hypoxia and fibrosis using magnetic resonance imaging. J Am Soc Nephrol 22(8):1429-1434. https://doi.org/10.1681/ASN. 2010111143

16. Xin-Long P, Jing-Xia X, Jian-Yu L, Song W, Xin-Kui T (2012) A preliminary study of blood-oxygen-level-dependent MRI in patients with chronic kidney disease. Magn Reson Imaging 30(3):330-335. https://doi.org/10.1016/j.mri.2011.10.003

17. Prasad PV, Thacker J, Li LP, et al. (2015) Multi-parametric evaluation of chronic kidney disease by MRI: a preliminary cross-sectional study. PloS ONE 10(10):e0139661. https://doi.org/10.1371/ journal.pone. 0139661

18. Nangaku M (2006) Chronic hypoxia and tubulointerstitial injury: a final common pathway to end-stage renal failure. J Am Soc Nephrol 17(1):17-25. https://doi.org/10.1681/ASN.2005070757

19. Michaely HJ, Metzger L, Haneder S, et al. (2012) Renal BOLDMRI does not reflect renal function in chronic kidney disease. Kidney Int 81(7):684-689. https://doi.org/10.1038/ki.2011.455

20. Kamper AL, Holstein-Rathlou NH, Strandgaard S, Leyssac PP, Munck O (1994) Glomerular filtration rate and segmental tubular function in the early phase after transplantation/uninephrectomy in recipients and their living-related kidney donors. Clin Sci 87(5):519-523

21. Khatir DS, Pedersen M, Jespersen B, Buus NH (2015) Evaluation of renal blood flow and oxygenation in CKD using magnetic resonance imaging. Am J Kidney Dis 66(3):402-411. https://doi.org/ 10.1053/j.ajkd.2014.11.022

22. Brezis M, Rosen S (1995) Hypoxia of the renal medulla-its implications for disease. N Engl J Med 332(10):647-655. https://doi.org/ 10.1056/NEJM199503093321006

23. Djamali A, Sadowski EA, Samaniego-Picota M, et al. (2006) Noninvasive assessment of early kidney allograft dysfunction by blood oxygen level-dependent magnetic resonance imaging. Transplantation 82(5):621-628. https://doi.org/10.1097/01.tp.0000 $234815.23630 .4 \mathrm{a}$ 